\title{
Steroid-insensitive tobacco smoke-induced lung inflammation models in the mouse
}

\author{
V Russell, D Spicer, P Woodman, A Connolly, A Young \\ From 2nd Cross Company Respiratory Symposium \\ Horsham, UK. 6-7 September 2012
}

\section{Background}

The effects of oral and inhaled steroids were investigated in murine models of lung inflammation induced by 4 or 11 days of exposure to tobacco smoke (TS). The effects were compared to other anti-inflammatory compounds.

\section{Methods}

Female mice were exposed daily to TS for 4 or 11 days. Control animals were exposed to air. Mice were killed $24 \mathrm{hrs}$ after the last TS-exposure, lungs lavaged and prepared for histological assessment. Steroids were given orally (dexamethasone (DEX) 300ug/ $/ \mathrm{kg}$; budesonide (BUD) $10 \mathrm{mg} / \mathrm{kg}, 1 \mathrm{hr}$ prior to, and $6 \mathrm{hrs}$ after each TS exposure) or intra-nasally (fluticasone proprionate (FP), DEX , BUD, beclomethasone, mometasone and ciclesonide dosed at $100-500 \mathrm{ug} / \mathrm{kg} 1 \mathrm{hr}$ prior to each TS-exposure). Steroid efficacy was also investigated in a mouse LPS lung inflammation model.

\section{Results}

TS-exposure induced a cellular infiltration into the lungs which was reproducible across more than 50 studies. The main cell types in the lavage were macrophages and neutrophils. Histopathological assessment showed a progressive increase in pathology from 4 days of exposure (predominantly vasculitis, alveolar congestion and epithelial hyperplasia) to 11 days of exposure (bronchiolitis, pneumonitis, bronchiolar degeneration, bronchial wall remodelling). Steroids dosed via the oral and intra-nasal routes failed to inhibit this inflammation in both models (maximal inhibition of TS-induced inflammation $14 \%$, all $\mathrm{p}>0.05$ compared to controls), although they caused body weight loss, confirming systemic availability and activity. The doses of steroids

used in the TS studies all showed significant efficacy in a mouse LPS-model (all $>65 \%$ inhibition, $\mathrm{p}<0.05$ ), confirming that the TS-exposure model was refractory to steroids. The TS-induced inflammation was reduced by PDE4 inhibitors such as Roflumilast when given either i.n. (total cells $50 \%$, neutrophils $60 \%$, both $\mathrm{p}<0.05$ ) or orally (total cells $50 \%$, neutrophils $66 \%$, both $\mathrm{p}<0.05$ ). Inhibitors of p38 MAP kinase were effective when given orally (BIRB796: total cells $42 \%$, neutrophils $55 \%$ inhibition, both $\mathrm{p}<0.05$ ) or i.n. (PF03715455: total cells 50\%, neutrophils $61 \%$ inhibition, both $\mathrm{p}<0.05)$. These inhibitory effects were confirmed in multiple studies where steroids continued to lack efficacy.

\section{Conclusions}

TS-exposure for up to 11 days induced lung inflammation. The inflammatory response was not affected by doses of steroids which were highly efficacious in a mouse LPS model. The steroid insensitive effects were robust and reproducible across multiple studies. The TS-induced inflammation was inhibited by a clinically used PDE4 inhibitor (Roflumilast) and p38 inhibitors such as PF03715455, which is currently in phase I clinical development.

Published: 14 August 2013

doi:10.1186/1476-9255-10-S1-P31

Cite this article as: Russell et al: Steroid-insensitive tobacco smoke-

induced lung inflammation models in the mouse. Journal of

Inflammation 2013 10(Suppl 1):P31.

Argenta Discovery, Spire Green, Harlow, Essex, UK

(c) 2013 Russell et al; licensee BioMed Central Ltd. This is an Open Access article distributed under the terms of the Creative Commons Attribution License (http://creativecommons.org/licenses/by/2.0), which permits unrestricted use, distribution, and reproduction in any medium, provided the original work is properly cited. 